www.revistadyo.com

\title{
Procedimiento para determinar el impacto de la gestión de riesgos en la sostenibilidad de las organizaciones
}

\author{
Yasmany Aguilera Sánchez, Juan Antonio Plasencia Soler y Fernando Marrero Delgado \\ https://doi.org/10.37610/dyo.v0i73.591
}

Recibido: 17 de Julio de 2020

Aceptado: 17 de Diciembre de 2020

\section{Resumen}

El procedimiento propuesto tiene como objetivo determinar el impacto de la gestión de riesgos en la sostenibilidad de las organizaciones mediante la construcción de un indicador integral partiendo del análisis de los riesgos, empleando el Proceso de Jerarquía Analítica (PJA). La investigación desarrolla un estudio de caso, método que permite su aplicación, partiendo de la identificación y análisis de los riesgos, se asocian a estos indicadores que posibilitan luego evaluar la sostenibilidad y definir un programa de mejoras que alcance a mitigar los riesgos logrando que la organización objeto de estudio incremente su nivel de sostenibilidad.

\section{Palabras clave}

Sostenibilidad; gestión de riesgos; indicador integral; proceso de jerarquía analítica.

\section{Introducción}

En el año 1987, el informe de la Comisión Mundial del Medio Ambiente, denominado "Nuestro Futuro Común", expone consideraciones sobre las estrategias ambientales a largo plazo, para lograr un desarrollo sostenible; definiendo este último como: "el desarrollo que satisface las necesidades de las presentes generaciones sin comprometer la capacidad de las futuras generaciones de satisfacer sus necesidades" (WCED, 1987).

En los últimos años y asociado a la necesidad de medir el comportamiento de una organización, y con auspicio de estándares internacionales, el concepto de desarrollo sostenible evolucionó hacia la sostenibilidad corporativa (SC). La SC tiene como objetivo aumentar o estabilizar el desempeño de la organización a través del tiempo, reduciendo los impactos negativos al medio ambiente, la obtención de ganancias y aportando valores a largo plazo, en términos financieros, sociales, ecológicos y éticos.

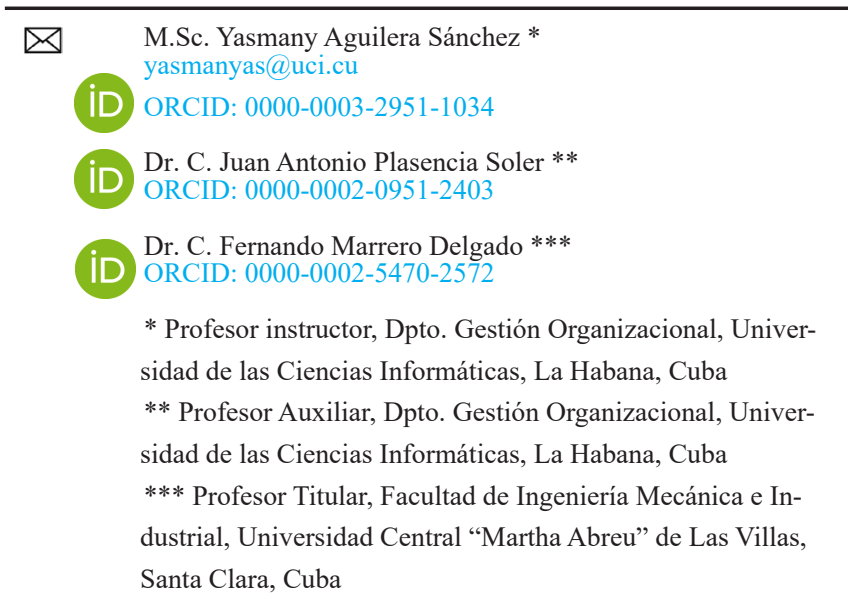

En la última década la sostenibilidad ha cobrado relevancia a la par del desarrollo de actividades y estrategias medioambientales. Estas prácticas permiten mostrar los avances que las empresas experimentan para alcanzar una producción menos contaminante del medioambiente y una gestión de sus procesos más responsable con la sociedad.

La gestión de riesgos (GR) de las organizaciones incluye los procesos relacionados con llevar a cabo la planificación, la identificación, el análisis, la planificación de respuesta a los riesgos, así como su monitoreo y control (AguileraSánchez, 2019). Un estudio realizado a 12 definiciones de GR en los últimos 10 años permitió identificar los términos o palabras claves que más se observan en los conceptos. El $92 \%$ de las definiciones estudiadas presentan enfoque de procesos; así como en un $83 \%$ hacen referencia a las fases de la gestión de riesgos de forma explícita, además, el 58\% hace referencia a las estrategias para el seguimiento de los riesgos. La definición emitida por (Bolaño-Rodríguez, 2014) cumple con todas estas características antes mencionadas; sin embargo es válido resaltar que la multidimensionalidad y el carácter ético, características fundamentales de la sostenibilidad, no se evidencian en ninguno de los conceptos.

Por lo anteriormente expuesto, se propone como definición de gestión de riesgos sostenible para la presente investigación la emitida por (Aguilera-Sánchez, 2019): proceso sistémico que inicia con el análisis estratégico de la organización para el desarrollo de un conjunto de actividades de prevención, diagnóstico, mitigación, control, evaluación de forma multidimensional y seguimiento; para minimizar los riesgos y sus efectos adversos, fomentando los positivos que se materializan en los procesos que afectan el cumplimiento de los objetivos, así como potenciar el carácter ético de la organización y la toma de decisiones en las acciones. 
Sobre esta base, los objetivos de la GR son aumentar la probabilidad y el impacto de los eventos positivos, y disminuir la probabilidad y el impacto de los eventos adversos (Project Management Institute, 2017); para lograrlo, debe existir una armonía entre la exposición a los riesgos y la respuesta a estos, según el costo de aceptarlos, evitarlos, transferirlos, mitigarlos, planear contingencias o incluso ignorarlos.

Una parte imprescindible que mantiene una relación directa con la Calidad de los resultados de las organizaciones lo constituye la gestión de los riesgos (Hosseini-Motlagh, Nematollahi, y Nouri, 2018).

Una de las nueva tendencia investigativa lo constituye la gestión de riesgos sostenible (Sustainability Risck Management - SRM); la cual, a decir de (Zarte M., Pechmann A., y Nunes I. L., 2019) aún es escasa y se centra fundamentalmente en la sostenibilidad de riesgo de cadenas de suministros. Esta práctica de la gestión de los riesgos empresariales que aborda los riesgos ambientales y de responsabilidad social (Wijethilake y Lama, 2018). Sin embargo, a decir de (Porras-Barajas, 2017) los estándares de la gestión de proyectos y las guías de los fundamentos de la gestión de proyectos no han sufrido la actualización que posibilite incorporar los elementos de la sostenibilidad, para migrar de la triple restricción alcance - tiempo - costo al concepto de triple resultado (Triple Bottom Line - TBL).

En el estudio realizado por (Chawla et al., 2018) concluyen que existen cuatro cambios que caracterizan la ventaja de la integración de la sostenibilidad y la gestión de riesgos en comparación con la gestión de riesgos clásica.

En primer lugar, un cambio de alcance: desde la gestión del tiempo, el presupuesto y la calidad hasta la gestión del impacto social, ambiental y económico. En segundo lugar, implica un cambio de paradigma: desde un enfoque que puede caracterizarse por la previsibilidad y el control, hasta un enfoque que se caracteriza por la flexibilidad, la complejidad y la oportunidad. En tercer lugar, consideran que la sostenibilidad implica un cambio de mentalidad para el gerente: desde la entrega de los resultados solicitados hasta la responsabilidad por el desarrollo sostenible en las organizaciones y la sociedad. (Chawla et al., 2018).

Por lo anteriormente expuesto, se define como objetivo desarrollar un procedimiento para determinar el impacto de la gestión de riesgos en la sostenibilidad de las organizaciones.

\section{Desarrollo}

Para la evaluación del impacto de la gestión de riesgos en la sostenibilidad de la organización se recomienda la construcción de un Indicador Integral para evaluar la Sostenibilidad (IIS) tal como se muestra en la figura 1.

\section{Paso 1. Identificación de riesgos}

Este procedimiento debe comenzar con el análisis de los resultados de la aplicación de acciones de control, auditorías; además se deben consultar el expediente de acciones de control internas y externas, así como la base de conocimientos; elemento que permitirá identificar los riesgos que en el histórico de la organización pueden tener un alto nivel de materialización en la actualidad.

Seguidamente, se deben identificar las causas que originan los riesgos, lo que permite garantizar que las acciones correctivas que se vayan a definir tengan la efectividad requerida; de manera que las acciones de mitigación del riesgo estén desarrolladas también en función de la causa que le dio origen. Al describir y registrar los riesgos, se debe utilizar un formato coherente para los enunciados de los riesgos con el objetivo de asegurar que cada riesgo sea de fácil entendimiento y no presente ambigüedad, para apoyar el desarrollo eficaz del análisis y de la respuesta a los mismos.
Figura 1 Procedimiento para determinar el impacto de la gestión de riesgos en la sostenibilidad de la organización.

Fuente: Elaboración propia

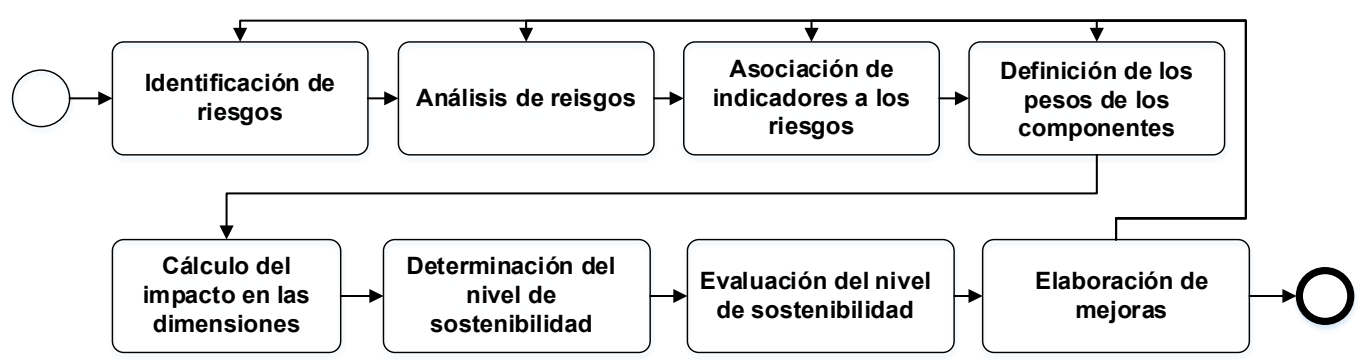


Identificar los riesgos es un proceso iterativo, ya que pueden surgir nuevos riesgos individuales de los proyectos, así como de la organización, a medida que el proyecto avanza.

\section{Paso 2. Análisis de riesgos}

Para la evaluación de los riesgos, se identifican los posibles impactos y probabilidad de ocurrencia. Se propone utilizar, un Mapa de riesgos; técnica que brindará mayor organización en el trabajo.

La principal modificación realizada y que constituye un aporte, a las investigaciones precedentes, es la evaluación del impacto del riesgo en las dimensiones de la sostenibilidad tal y como muestra la ecuación [6].

$$
\mathrm{Ic}_{\mathrm{i}}=\mathrm{P}_{\mathrm{i}}^{*} \operatorname{Imax}_{\mathrm{i}}^{*} \mathrm{D}_{\mathrm{i}}
$$

Donde:

$\mathrm{P}_{\mathrm{i}}$ : Probabilidad de ocurrencia del riesgo (i).

Imax $_{i}$ : Valor máximo del impacto luego de evaluar el riesgo (i) en las dimensiones de acuerdo al modelo de sostenibilidad adoptado (para un 3BL económica, social, ecológica).

$\mathrm{D}_{\mathrm{i}}$ : Nivel de detención.

Posteriormente, se clarifican los riesgos; en el que se propone utilizar el diagrama de Pareto. Este diagrama constituye un sencillo método de análisis que mediante un gráfico permite discriminar entre las causas más importantes de un problema (los pocos y vitales) y las que lo son menos importantes (los muchos y triviales).

\section{Paso 3. Asociación de indicadores a los riesgos}

Para realizar la asociación de indicadores a los riesgos se analizan los informes estadísticos de la organización, lo que permite identificar posibles indicadores que describan materialización de los riesgos. En este proceso se logra identificar de forma cuantitativa la materialización de los riesgos dentro de la organización.

En este paso, además se propone conformar una jerarquía teniendo como meta el nivel de sostenibilidad (Ns), al cual se asocian las dimensiones (Dimk), los Objetivos de control (Objci), los Riesgos (Ri) y los indicadores (Indh) asociados a los riesgos identificados, tal y como se muestra en la ecuación [2]:

$$
N s=N s(\operatorname{Dim}(\operatorname{Obc}(R(\operatorname{Ind}))))
$$

\section{Paso 4. Definición de los pesos de los componentes}

Luego de construida la jerarquía, se propone para la determinación de los pesos de cada componente, utilizar el Proceso de la Jerarquía Analítica (PJA) del idioma inglés "Analytic Hierarchy Process" (AHP) desarrollado por Tomas L. Saaty en la década de los setenta del siglo pasado. El PJA asigna un peso a los criterios a través de la construcción de una matriz de comparación por pares, donde los expertos, mediante una escala numérica, indican cuántas veces un elemento es más importante que otro ( Saaty, 2008; Saaty y Sagir, 2015 Jayawickrama, Kulatunga, y Mathavan, 2017).

Aunque los autores de la investigación sugiere este método subjetivo, debido a que permite evaluar la consistencia de los expertos en sus valoraciones a través del Coeficiente de Inconsistencia (CI); existen otros métodos subjetivos y objetivos que pueden ser utilizados para realizar el cálculo de la importancia relativa de los componentes que intervienen en la jerarquía definida (Romero-Gélvez, Cortes-Aldana, y Franco-Sepúlveda, 2015). Se recomienda en esta fase utilizar el software "Super Decisions" desarrollado por Tomas L. Saaty para una mayor agilidad y fiabilidad en el cálculo de los pesos.

\section{Paso 5. Cálculo del impacto en las dimensiones}

En este paso se parte del valor de la evaluación de los indicadores y de su valor ideal definido en el catálogo de indicadores. Por la naturaleza diversa de los valores que asumen los indicadores, estos deben ser normalizados y homogenizados, por lo que el autor sugiere, utilizar el método de normalización de distancia a la meta utilizado por varios investigadores en los últimos años (Oppio, Corsi, Torrieri, y Mattia, 2017; Plasencia-Soler, J. A., Marrero-Delgado, F., Nicado-García, M. y Aguilera-Sánchez, Y 2017; Xuan Zuo, Hui Hua, Zhanfeng Dong, y Chunxu Hao, 2017). Para los valores que se busca maximizar el resultado se utiliza la ecuación [3]:

$$
N_{h i j k}=\frac{V r_{h i j k}}{V i_{h i j k}}
$$

Para valores que se relacionen con indicadores en los que se busca minimizar el resultado se utiliza la ecuación [4]:

$$
N_{h i j k}=\frac{V i_{h i j k}}{V r_{h i j k}}
$$

Donde:

$\mathbf{N}_{\text {hijk: }}$ : Vector normalizado del indicador (h) asociado al riesgo (i) del Objc (j) de la dimensión (k).

$\mathbf{V i}_{\mathrm{ijk}}$ : Valor ideal del indicador (h) asociado al riesgo (i) del Objc (j) de la dimensión (k). 
$\mathbf{V r}_{\text {jik }}$ : Valor real del indicador (h) asociado al riesgo (i) del Objc (j) de la dimensión (k).

Luego se calcula el impacto de las dimensiones $\left(\mathbf{D i m}_{\mathrm{k}}\right)$, como se muestra en la ecuación [5]:

$$
\operatorname{Dim}_{k}=\sum_{j=1}^{n} O b j c_{j k} * W_{j k}
$$

Para $i=1 \ldots n$

Dónde:

Objc $_{\mathrm{jk}}$ : Objetivo de control $\mathrm{j}$ asociado a la dimensión $\mathrm{k}$.

$\mathrm{W}_{\mathrm{jk}}$ : Peso del Objc (j) asociado a la dimensión $\mathrm{k}$.

Para determinar los Objc (j) se utiliza la ecuación [6]:

$$
O b j c_{j k}=\sum_{i=1}^{n} R_{i j k} * W_{i j k}
$$

Para $\mathrm{i}=1 \ldots \mathrm{n}$

Dónde: $\mathrm{W}_{\mathrm{ijk}}$ peso del riesgo (i) asociado al Objc (j) de la dimensión $(\mathrm{k})$.

Para determinar los R(i) se utiliza la ecuación [7]:

$$
R_{i j k}=\sum_{h=1}^{n} N_{h i j k k} * W_{h i j k}
$$

Para $i=1 \ldots n$

Donde: $\mathrm{W}_{\mathrm{jik}}$ peso del indicador asociado al riesgo (j) asociado al Objc (i) de la dimensión (k).

\section{Paso 6. Determinación del coeficiente de sostenibilidad}

Luego se procede a determinar el coeficiente de sostenibilidad (Cs) de la organización teniendo en cuenta el impacto de las dimensiones como se muestra en la ecuación [8]:

\section{Tabla 1 Escala de}

evaluación del nivel de sostenibilidad.

Fuente: (Plasencia-Soler, J. A., 2018).

$$
\text { Cs }=\sum_{k=1}^{n} \operatorname{Dim}_{k} * W_{k}
$$

Para $i=1 \ldots n$

Donde: $\mathrm{W}_{\mathrm{k}}$ peso asociado a la dimensión $(\mathrm{k})$.

Los valores de las variables que componen la jerarquía y los pesos se expresan, luego de normalizarse, en valores entre 0 y 1 , entonces el coeficiente de sostenibilidad (Cs) es evaluado entre 0 y 1 en dependencia del comportamiento sostenible de la organización. Mientras más se acerque el valor Cs al valor de uno, mayor será el nivel de sostenibilidad.

\section{Paso 7. Evaluación del nivel de sostenibilidad}

En este paso, se propone evaluar el coeficiente calculado a través de una escala de valoración ordinal, donde a cada valor de (Cs), se le asigna una etiqueta, que corresponde a un nivel de sostenibilidad (Ns). De acuerdo con (Medina-León et al., 2014) generalmente este tipo de escala se utiliza para determinar el estado de la organización objeto de estudio.

Varios son los métodos para determinar el rango de valores de la escala para evaluar sostenibilidad, sin embargo uno de los más utilizados según (Medel-González, García-Ávila, Salomon, Marx-Gómez, y Hernández, 2016) lo constituye la consulta a expertos.

Por otra parte, la cantidad de intervalos varía entre tres y nueve, por ejemplo, la Ethos (2014) establece cinco estados para evaluar los indicadores asociados a la sostenibilidad, mientras la UNESCO (2006) propone nueve categorías para el mismo fin.

Teniendo en cuenta los referentes anteriores y utilizando la consulta con expertos, permitió mejorar paulatinamente la escala, de manera que refleje las características del objeto de estudio. Se propone la escala de valores para determinar el nivel de sostenibilidad organizacional (Tabla 1)

\begin{tabular}{|l|l|}
\hline $\begin{array}{l}\text { Coeficiente de } \\
\text { sostenibilidad } \\
(\boldsymbol{C s})\end{array}$ & $\begin{array}{l}\text { Nivel de sostenibilidad } \\
(\boldsymbol{N S})\end{array}$ \\
\hline $0.90 \leq C s \leq 1$ & Muy alto \\
\hline $0.75 \leq C s \leq 0.89$ & Alto \\
\hline $0.50 \leq C s \leq 0.74$ & Medio \\
\hline $0.35 \leq C s \leq 0.49$ & Bajo \\
\hline $0.00 \leq C s \leq 0.34$ & Muy bajo \\
\hline
\end{tabular}




\section{Paso 8. Elaboración de programa de mejoras}

En este paso las fuentes fundamentales de las mejoras lo constituyen los resultados de los valores de los indicadores cuantitativos definidos y los índices de sostenibilidad alcanzados en la evaluación.

Esta fase concluye con la elaboración de un plan de acciones de mejoras, donde se integren las propuestas de los cinco pasos. Este plan debe contener, entre otros aspectos: riesgo, acción de mejora, responsable de la acción, fecha de cumplimiento, impacto esperado de la mejora, además de indicar a que dimensión y proceso corresponde.

\section{Resultados}

El presente procedimiento como parte de un estudio de caso fue aplicado en el Centro de Ideoinformática (CIDI). Centro de desarrollo de software que se encarga de proveer soluciones integrales, productos y servicios relacionados con las tecnologías de internet, en función de la defensa de la ideología socialista. La organización cuenta con 98 trabajadores, de los cuales 72 son especialistas vinculados a la producción de software. Para la aplicación del procedimiento propuesto fueron analizados los resultados de los instrumentos aplicados, así como de los informes de acciones de control ejecutadas en el área a cada uno de los procesos claves de la organización, unido al estudio del plan de prevención de riesgos, el equipo de expertos conformado por siete especialistas, consideró que los riesgos son los que se recogen en la (Tabla 2).

Para identificar los expertos se tuvieron en cuenta, la experiencia profesional en relación con el objeto de investigación, la participación en investigaciones relacionadas con esta temática, el dominio teórico de la temática, la preparación académica y científica, y la experiencia; de modo que estuvieran en capacidad de ofrecer valoraciones y hacer recomendaciones pertinentes. Para la aplicación del método se identificaron 13 posibles expertos de los que fueron seleccionados 7. De ellos 5 son doctores y 2 másteres en ciencias y todos se encuentran vinculados al área de gestión de riesgos.

Una vez identificados los riesgos y sus posibles manifestaciones asociadas a estos, se procede a realizar la evaluación de los riesgos, lo que propició utilizar la técnica de Pareto para ordenar los mismos. El ordenamiento del ejemplo anterior queda de la forma siguiente: R12; R04; R03; R13; R09; R11; R14; R01; R10; R08; R15; R05; R06; R07 y R02.

A cada uno de los riesgos se les asocia una o varias causas que pueden propiciar la materialización de los mismos.

\begin{tabular}{|c|c|c|c|}
\hline \multirow{13}{*}{$\begin{array}{l}\text { Tabla } 2 \text { Listado de } \\
\text { riesgos y sus posibles } \\
\text { manifestaciones. } \\
\text { Fuente: Elaboración } \\
\text { propia. }\end{array}$} & Código & Riesgo & Manifestación \\
\hline & R01 & $\begin{array}{l}\text { Ocurrencia de indisciplinas, ilegalidades y actos de } \\
\text { corrupción }\end{array}$ & Actitudes inadecuadas como el favoritismo, nepotismo y corrupción \\
\hline & R02 & Afectación en la transportación obrera & Incremento del índice de ausentismo \\
\hline & & Sobreconsumo innecesario de portadores eneróticos & Deterioro de los índices de consumo de los portadores. \\
\hline & & & Despilfarro de portadores energéticos. \\
\hline & & & Ocurrencia de incendios \\
\hline & & & Accidentes de trabajo. \\
\hline & & & Bajo índice de publicaciones científicas \\
\hline & R05 & 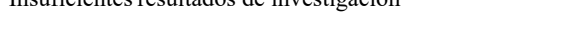 & Bajo impacto de las investigaciones \\
\hline & & & Navegación por sitios no permitidos por su contenido \\
\hline & R06 & Uso indebido de los servicios tecnológicos y telemáticos & Uso indebido de contraseñas y desvío de claves de administración \\
\hline & R07 & Apropiación indebida de los recursos para uso personal & Desvío de recursos tecnológicos \\
\hline & R08 & Incumplimiento de contrato & Baja calidad en el desarrollo del software \\
\hline
\end{tabular}




\begin{tabular}{|c|c|c|}
\hline Código & Riesgo & Manifestación \\
\hline \multirow{2}{*}{ R09 } & \multirow{2}{*}{ Pérdida de patrimonio } & Robo de ideas \\
\hline & & Pérdida de código fuente \\
\hline $\mathrm{R} 10$ & Pérdida de clientes & Incumplimiento de los planes de ingresos \\
\hline R11 & Pérdida de prestigio & $\begin{array}{l}\text { Excesos en las relaciones de trabajo y negociaciones con clientes } \\
\text { nacionales y/o extranjeros }\end{array}$ \\
\hline $\mathrm{R} 12$ & $\begin{array}{l}\text { Faltantes y sobrantes de Activos Fijos Tangibles } \\
\text { (AFT) }\end{array}$ & Desactualización de la cuenta de AFT \\
\hline R13 & Afectación económica al presupuesto del Estado & Pago indebido a trabajadores \\
\hline \multirow{3}{*}{ R14 } & \multirow{3}{*}{$\begin{array}{l}\text { Pérdida o extravío de Información oficial clasificada } \\
\text { (IOC) }\end{array}$} & Generar IOC sin identificar como tal \\
\hline & & Clasificar la información oficial incorrectamente \\
\hline & & $\begin{array}{l}\text { Destruir o borrar IOC sin la autorización correspondiente y sin el } \\
\text { debido control }\end{array}$ \\
\hline $\mathrm{R} 15$ & Violación del principio de compartimentación & $\begin{array}{l}\text { Acceso por personas sin autorización a IOC en las computadoras } \\
\text { establecidas para ello }\end{array}$ \\
\hline
\end{tabular}

Luego del análisis de los riesgos, se procede a definir los objetivos de control del área, los que permitirán demostrar el resultado o propósito que se desea alcanzar. Estos se obtienen de la vinculación de los riesgos identificados con los objetivos estratégicos de la organización; además se clasifican por dimensiones de la sostenibilidad para en una etapa posterior poder cuantificar su impacto. Los riesgos, objetivos de control y dimensiones definidas se recogen en la (Tabla 3).

Tabla 3 Riesgos con sus objetivos de control y dimensiones a las que tributan.

Fuente: Elaboración propia.

\begin{tabular}{|c|c|c|}
\hline Código & Objetivos de control & Dimensión \\
\hline R01 & \multirow[t]{2}{*}{$\begin{array}{l}\text { Garantizar que los trabajadores se distingan por una sólida preparación técnica y profesional, } \\
\text { ejemplaridad, principios éticos, políticas e ideológicas }\end{array}$} & \multirow[t]{2}{*}{ Social } \\
\hline R02 & & \\
\hline R03 & $\begin{array}{l}\text { Implementar un sistema que posibilite el control de los portadores energéticos impactando positivamente } \\
\text { en el cuidado del medioambiente }\end{array}$ & Ecológica \\
\hline R04 & $\begin{array}{l}\text { Garantizar una cultura de seguridad y salud del trabajo que permita minimizar la ocurrencia de accidentes } \\
\text { de trabajo y la pérdida de vidas humanas. }\end{array}$ & \multirow{4}{*}{ Social } \\
\hline R05 & $\begin{array}{l}\text { Estructurar la investigación y el postgrado en estrecha relación con las líneas, grupos y proyectos de } \\
\text { investigación, desarrollo e innovación }\end{array}$ & \\
\hline R06 & \multirow[t]{2}{*}{$\begin{array}{l}\text { Fortalecer la infraestructura tecnológica que respalda los procesos sustantivos garantizando la } \\
\text { seguridad de la información }\end{array}$} & \\
\hline R07 & & \\
\hline
\end{tabular}




\begin{tabular}{|c|c|c|}
\hline Código & \multicolumn{1}{|c|}{ Objetivos de control } & Dimensión \\
\hline \hline R08 & \multirow{2}{*}{$\begin{array}{l}\text { Producir aplicaciones y servicios informáticos sirviendo de soporte a la industria cubana del } \\
\text { software }\end{array}$} & Económica \\
\hline R09 & $\begin{array}{l}\text { L10 } \\
\text { Lograr un control efectivo en cada uno de los subsistemas establecidos, de forma tal que garantice la } \\
\text { confiabilidad de la contabilidad }\end{array}$ & \\
\hline R11 & $\begin{array}{l}\text { Lograr un adecuado sistema de protección de la información oficial clasificada (IOC) que garantice la } \\
\text { correcta gestión de la misma }\end{array}$ & Social \\
\hline R13 & & \\
\hline R14 & & \\
\hline
\end{tabular}

Se aplicó el Método de PJA de Saaty para el cálculo de los pesos de cada uno de los elementos que componen la jerarquía analítica definida. Los expertos realizaron la asignación de los pesos por consenso, a través de comparaciones pareadas de cada una de las dimensiones, Objetivos de control, riesgos e indicadores de la jerarquía.
Luego, son calculados los valores de impactos por cada Riesgo, Objetivo de control, las dimensiones de la sostenibilidad y finalmente el coeficiente de sostenibilidad de la organización, el cual para el cierre del mes de julio del 2019 ofrece un valor de 0.47 utilizando el catálogo de indicadores que se recoge en la (Tabla 4). Este valor evaluado en la escala propuesta, ofrece como resultado un nivel de sostenibilidad Medio $(0,47 \leq \mathrm{Ne} \leq 0,62)$.

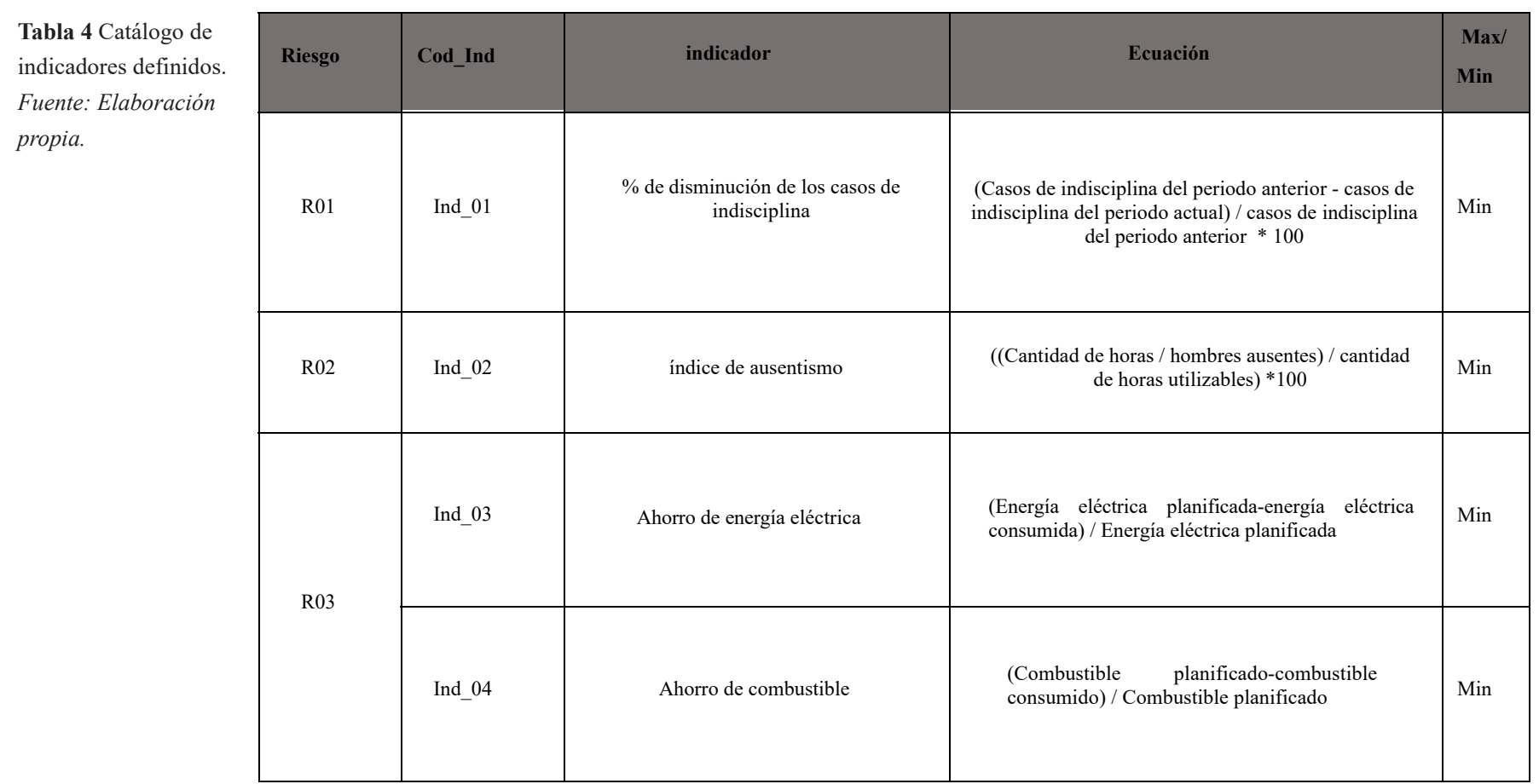




\begin{tabular}{|c|c|c|c|c|}
\hline Riesgo & Cod_Ind & indicador & Ecuación & $\begin{array}{l}\operatorname{Max} / \\
\operatorname{Min}\end{array}$ \\
\hline \multirow[b]{2}{*}{ R04 } & Ind_05 & Índice de trabajadores fallecidos & $\begin{array}{l}\text { (Cantidad de trabajadores fallecidos/total de } \\
\text { trabajadores) } * 100\end{array}$ & Min \\
\hline & Ind_06 & Índice de trabajadores accidentados & $\begin{array}{l}\text { (Cantidad de trabajadores accidentados / } \\
\text { total de trabajadores) } * 100\end{array}$ & Min \\
\hline R05 & Ind_07 & $\begin{array}{l}\text { Índice de publicaciones grupos I y II por } \\
\text { profesor y especialistas }\end{array}$ & $\begin{array}{l}\text { (Cantidad de publicaciones grupos I y II / total de } \\
\text { profesores y especialistas) } * 100\end{array}$ & $\operatorname{Max}$ \\
\hline R06 & Ind_08 & $\begin{array}{l}\text { índice de hechos de uso indebido de } \\
\text { servicios tecnológicos y telemáticos }\end{array}$ & (Cantidad hechos / total de hechos) $* 100$ & Min \\
\hline R07 & Ind_09 & $\begin{array}{l}\text { índice de hechos de apropiación de } \\
\text { recursos }\end{array}$ & (Cantidad hechos / total de hechos) $* 100$ & Min \\
\hline R08 & Ind_10 & $\begin{array}{l}\text { Índice de actividades pactadas } \\
\text { incumplidas }\end{array}$ & $\begin{array}{l}\text { (Cantidad de actividades incumplidas / } \\
\text { total de actividades) } * 100\end{array}$ & Min \\
\hline R09 & Ind_11 & Índice de investigaciones patentadas & $\begin{array}{l}\text { (Cantidad de investigaciones patentadas / total de } \\
\text { investigaciones) } * 100\end{array}$ & $\operatorname{Max}$ \\
\hline \multirow{2}{*}{ R10 } & Ind_12 & $\%$ de retención del cliente & (Cantidad de clientes retenidos / total de clientes) $* 100$ & $\operatorname{Max}$ \\
\hline & Ind_13 & $\%$ de incremento de clientes & $\begin{array}{c}\text { (Clientes del periodo actual - clientes del periodo } \\
\text { anterior) } / \\
\text { (Clientes del periodo actual) } * 100\end{array}$ & $\operatorname{Max}$ \\
\hline R11 & Ind_14 & $\begin{array}{l}\text { Índice de proyectos cerrados } \\
\text { insatisfactoriamente }\end{array}$ & $\begin{array}{l}\text { (Proyectos cerrados insatisfactoriamente / total de } \\
\text { proyectos) } * 100\end{array}$ & Min \\
\hline R12 & Ind_15 & $\begin{array}{l}\text { Índice de Activos Fijos Tangibles } \\
\text { (AFT) declarados como sobrantes o } \\
\text { faltantes }\end{array}$ & $\begin{array}{c}\text { ((Cantidad AFT sobrantes + cantidad AFT faltantes }) / \\
\text { total de AFT }) * 100\end{array}$ & Min \\
\hline R13 & Ind_16 & $\begin{array}{l}\text { Variación en el } \\
\text { Presupuesto }\end{array}$ & (Presupuesto real / presupuesto plan) $* 100$ & Min \\
\hline R14 & Ind_17 & $\begin{array}{l}\text { Índice de hechos de pérdida o } \\
\text { extravío de Información Oficial } \\
\text { Clasificada (IOC) }\end{array}$ & (Cantidad hechos / total de hechos) $* 100$ & Min \\
\hline R15 & Ind_18 & $\begin{array}{l}\text { Índice de violaciones de } \\
\text { compartimentación }\end{array}$ & (Cantidad hechos / total de hechos) $* 100$ & Min \\
\hline
\end{tabular}

Seguidamente, se pone en práctica el plan de mejoras que se recoge en la (Tabla 5), lo que posibilitó contribuir a mejorar los indicadores asociados a la jerarquía para una futura reevaluación.

Posteriormente se realiza una reevaluación del nivel de sostenibilidad de la organización objeto de estudio al cierre de enero 2020 utilizando los mismos indicadores, para comprobar la contribución a la sostenibilidad del procedimiento. En este caso se logra constatar que el coeficiente de sostenibilidad es de 0.78 ; el cual ofrece como resultado un nivel de sostenibilidad Alto $(0.75 \leq \mathrm{Cs} \leq 0.89)$. 
Tabla 5 Programa para la mitigación de riesgos. Fuente: Elaboración propia.

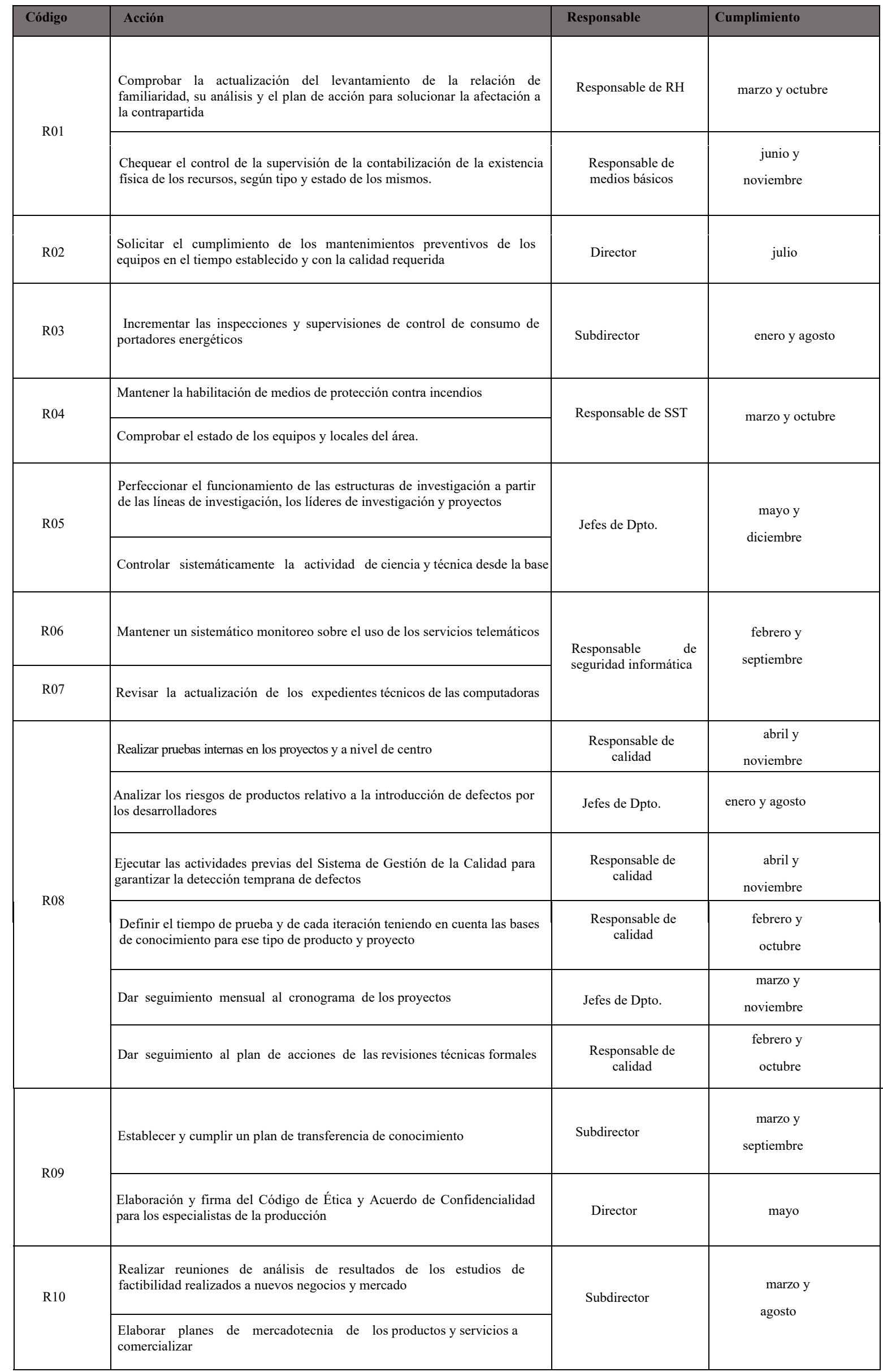




\begin{tabular}{|c|c|c|c|}
\hline Código & Acción & Responsable & Cumplimiento \\
\hline \multirow{2}{*}{ R11 } & $\begin{array}{l}\text { Seguimiento por parte de la Dirección de las actividades a desarrollar con } \\
\text { cada cliente }\end{array}$ & Director & marzo, julio y diciembre \\
\hline & $\begin{array}{l}\text { Rotación de los profesionales por los diferentes sectores de la economía en } \\
\text { que se encuentra dividido el mercado. }\end{array}$ & Director & junio \\
\hline \multirow{2}{*}{$\mathrm{R} 12$} & $\begin{array}{l}\text { Conciliación sistemática con la dirección de contabilidad y finanzas del } \\
\text { submayor de AFT }\end{array}$ & \multirow{2}{*}{$\begin{array}{l}\text { Responsable de } \\
\text { medios básicos }\end{array}$} & \multirow{2}{*}{$\begin{array}{r}\text { enero y } \\
\text { septiembre }\end{array}$} \\
\hline & $\begin{array}{l}\text { Verificar que aparezca el estado real de los AFT en los informes de las } \\
\text { entregas de cargos }\end{array}$ & & \\
\hline R13 & $\begin{array}{l}\text { Verificar sistemáticamente el cumplimiento del procedimiento para la } \\
\text { confección de la prenómina }\end{array}$ & Subdirector & abril y octubre \\
\hline \multirow{2}{*}{ R14 } & $\begin{array}{l}\text { Determinar mediante el uso correcto de la Lista Interna la información que } \\
\text { deba ser clasificada Identificar los responsables de la custodia y } \\
\text { clasificación de la IOC }\end{array}$ & \multirow{2}{*}{ Director } & \multirow{2}{*}{ mayo y agosto } \\
\hline & $\begin{array}{l}\text { Capacitar al personal encargado de manejar IOC con el procedimiento } \\
\text { establecido para la destrucción de este tipo de información }\end{array}$ & & \\
\hline $\mathrm{R} 15$ & $\begin{array}{l}\text { Chequear la identificación de los medios computacionales en los que se } \\
\text { gestiona la IOC }\end{array}$ & $\begin{array}{l}\text { Responsable de } \\
\text { seguridad informática }\end{array}$ & febrero \\
\hline
\end{tabular}

\section{Conclusiones}

El procedimiento general, así como los métodos, técnicas y herramientas que lo integran, constituyen una alternativa viable para contribuir a la sostenibilidad desde la gestión riesgos; al tener como referencia las teorías, estándares internacionales y tendencias modernas para el desarrollo sostenible, así como las características del entorno y marco regulatorio; lo que contribuye a la gestión, evaluación y mejora de la sostenibilidad organizacional.

Evaluar el impacto de la gestión de riesgos en la sostenibilidad de una organización, a través de un indicador integral estructurado en dimensiones, objetivos de control, riesgos e indicadores; permite identificar mejoras potenciales en la toma de decisiones.

Los resultados obtenidos de la aplicación del procedimiento en una entidades del sector de las Tecnologías de la Información y las Comunicaciones en la provincia La Habana, permitió identificar las dimensiones y variables que más afectaban su desempeño sostenible, siendo la base para la elaboración de objetivos y trazar líneas de accion que contribuyeron a evaluar y mejorar los niveles de sostenibilidad.
El estudio de caso evidenció la posibilidad de aplicación del procedimiento, cuyos resultados fueron favorables al contribuir a la sostenibilidad de la organización; evidenciándose en el incremento de la sostenibilidad de un 0.47 a 0.78 .

Al abordar la relación entre la gestión de riesgos y la sostenibilidad, esta investigación, expone una base metodológica para futuros estudios donde se vinculen otras formas de gestión empresarial al desarrollo sostenible de las organizaciones.

\section{Referencias bibliográficas}

AGUILERA-SÁNCHEZ Y. (2019). Gestión de riesgos para contribuir a la sostenibilidad de las organizaciones orientadas a proyectos (Tesis de Maestría). Universidad de las Ciencias Informáticas, La Habana, Cuba.

BOLAÑO-RODRÍGUEZ Y. (2014). Modelo de dirección estratégica basado en la administración de riesgos para la integración del sistema de dirección de la empresa (Tesis Doctoral). Universidad Tecnológica de la Habana José Antonio Echeverría, La Habana. Cuba. 
CHAWLA, V., CHANDA, A., ANGRA, S., y CHAWLA, G. (2018). "The sustainable project management: A review and future possibilities». Journal of Project Management, 3(3), pp. 157-170.

HOSSEINI-MOTLAGH S.-M., NEMATOLLAHI M., y NOURI M. (2018). «Coordination of green quality and green warranty decisions in a two-echelon competitive supply chain with substitutable products». Journal of Cleaner Production, 196, pp. 961-984.

https://doi.org/10.1016/j.jclepro.2018.06.123

JAYAWICKRAMA, H. M. M. M., KULATUNGA, A. K., y MATHAVAN, S. (2017). «Fuzzy AHP based Plant Sustainability Evaluation Method». Procedia Manufacturing, 8 (Supplement C), pp. 571-578. https://doi.org/10.1016/j.promfg.2017.02.073

MEDEL-GONZÁLEZ, F., GARCÍA-ÁVILA, L. F., SALOMON, V. A. P., MARX-GÓMEZ, J., Y HERNÁNDEZ, C. T. (2016). «Sustainability performance measurement with Analytic Network Process and balanced scorecard: Cuban practical case». Production, 26(3), pp. 527-539.

https://doi.org/10.1590/0103-6513.189315

MEDINA-LEÓN, A., RICARDO-ALONSO, A., PILOTO-FLEITAS, N., NOGUEIRA-RIVERA, D., HERNÁNDEZ-NARIÑO, A., Y CUÉTARASÁNCHEZ, L. (2014). «Índices integrales para el control de gestión: consideraciones y fundamentación teórica». Ingeniería Industrial, 35(1), pp. 94-104.

OPPIO A., CORSI S., TORRIERI F., y MATTIA S. (2017). «Infrastructure Development and Territorial Vulnerability. The Role of Composite Indicators for Addressing Siting Decisions». From Theory to Practice. pp. $277-290$

https://doi.org/10.1007/978-3-319-49676-4_21

PLASENCIA-SOLER J. A. (2018). Modelo para contribuir a la sostenibilidad de entidades de las tecnologías de la información y las comunicaciones desde la gestión estratégica (Tesis Doctoral). UCI, La Habana, Cuba.

PLASENCIA-SOLER, J. A., MARRERO-DELGADO, F., NICADO-GARCÍA, M. y AGUILERA-SÁNCHEZ, Y. (2017). «Procedimiento para la priorización de factores críticos de éxito». DYNA, 84(202), pp. 26-34. DOI: http://dx.doi.org/10.15446/dyna.v84n202.62084.
PORRAS-BARAJAS N. (2017). «Una mirada a la sostenibilidad en la gestión de proyectos». Daena: International Journal of Good Conscience, 12(3), pp. 328-344.

PROJECT MANAGEMENT INSTITUTE. (2017). A Guide to the Project Management Body Knowledge (PMBOK Guide 6th Edition). Recuperado a partir de https:/www.pmi.org/pmbok-guide-standards/ foundational/pmbok

ROMERO-GÉLVEZ, J. I., CORTES-ALDANA, F. A., y FRANCO-SEPÚLVEDA, G. (2015). «Compromise solutions in mining method selection - case study in colombian coal mining». DYNA, 82(191), pp. 127-136

SAATY, T. L. (2008). «Decision making with the analytic hierarchy process». International journal of services sciences, 1(1), pp. 83-98. https://doi.org/10.1504/ ijssci.2008.017590

SAATY, T. L. y SAGIR, M. (2015). «Ranking countries more reliably in the summer olympics». International Journal of the Analytic Hierarchy Process, 7(3), pp. 589- 610. https://doi.org/10.13033/ijahp.v7i3.341

WIJETHILAKE, C. y LAMA, T. (2019). «Sustainability core values and sustainability risk management: Moderating effects of top management commitment and stakeholder pressure». Business Strategy and the Environment, 28(1), pp. 143-154.

DOI: https://doi. org/10.1002/bse.2245

WCED. (1987). Our Common Future. United Nations. Recuperado a partir de http://www.un-documents.net/ wced-ocf.htmm

XUAN ZUO, HUI HUA, ZHANFENG DONG, y CHUNXU HAO. (2017). «Environmental Performance Index at the Provincial Level for China 2006-2011». Ecological Indicators, 75, pp. 48-56. https://doi.org/10.1016/ j.ecolind.2016.12.016

ZARTE M., PECHMANN A., y NUNES I. L. (2019). «Decision support systems for sustainable manufacturing surrounding the product and production life cycle A literature review». Journal of Cleaner Production, vol. 219, pp. 336-349, DOI: https://doi.org/10.1016/j. jclepro.2019.02.092 